\title{
THE EVOLUTION OF SYNTACTIC CAPACITY FROM NAVIGATIONAL ABILITY
}

\author{
MARK BARTLETT \& DIMITAR KAZAKOV \\ Department of Computer Science, University of York, \\ Heslington, York, YO10 5DD, UK
}

\section{Syntax And Navigation}

Many recent computational models (most notably those of Kirby (2002)) have shown how syntax may naturally emerge in language in order to exploit structural properties of a semantic space. However, while such models can explain why early human protolanguages may have gained in structural complexity to become full languages, they do not explain how the ability of individuals to handle compositionality of linguistic fragments evolved: while existing models explain the emergence of syntax in language, this is predicated on an existing syntax handling capability. We present one possible explanation for the evolution of this neurological under-pinning of syntax, and outline results from a computational model which has been developed to assess its feasibility.

We believe a link exists between motor and verbal sequence processing that may hold the key to the origins of syntax. We have previously discussed a model of navigation which demonstrates this link (Kazakov \& Bartlett 2004), using landmarks as beacons and describing the path between two points by the list of landmarks one has to pass by on a journey from one position to another. One can devise a impoverished formalisation which represents such a map as a regular grammar, in which landmarks correspond to terminals, crossroads to non-terminals, and rules describe paths between two positions, e.g. the rule $Y \rightarrow$ $X l_{1} l_{2} l_{3}$ states that to reach $Y$ it is sufficient to be at $X$ and then to pass by the three landmarks listed in order. With this representation, planning or following a path is equivalent to generating or parsing, respectively, a sentence of a regular language (RL). Should the navigational needs of individuals necessitate return along the same path as the outward journey, the navigational task requires a more complex formulation equivalent to a context-free language (CFL). The equivalence between the processor needed to understand these routes and a RL or CFL parser is important: if a parser was needed for navigation, it may have first evolved for this purpose. Once this parser was developed, only a relatively small change in the neural connections may have been required to make this parser available to the human brain speech circuitry.

This theory draws support from existing neurological research. Ullman (2004) pinpoints several memory circuits in the brain, the procedural memory, which 
are associated with syntactic processing, and are distinct from declarative memory which stores information about facts and events, including the mental lexicon. The model suggests a common basis for the processing of verbal and non-verbal sequences which is supported by others, such as Hoen et al.(2003) who report that using non-verbal symbols to exercise the ability to reorder sequences, helps patients with speech difficulties to understand sentence that need to have their constituents rearranged in the same way (such as to form a passive sentence).

\section{Evidence From Artificial Life}

In order to test the evolutionary plausibility of this theory to explain the origins of linguistic syntactic ability, a second, supplemental theory, that one of the original purposes of language may have been for use in navigation, has been developed. From this, a multi-agent simulation has been created in which the relative performance of populations with differing behaviours are tested for their abilities to survive and reproduce. The behaviours in the model incorporate varying degrees of planning/parsing competence and those linguistic and navigational activities possible at each level. Experimental results indicate clear advantages, as manifested by greater population sizes, in those populations in which communication is permitted, especially when 'syntactic' navigation is used. In addition to using the model to essay the relative successes of these behaviours, the role of the environment structure in determining the benefit of a behaviour has also been examined. It has been established that populations able to communicate grow faster and are more resilient to volatility of resources than those unable to do so. Such results point towards a possible source of evolutionary pressure for the ability to use language. This, combined with the biological plausibility of adapting navigational abilities into syntactic handling skills for language, suggests that this theory be further considered as one possible mechanism to explain the origins of syntactic ability in humans.

\section{References}

Hoen, M., Golembiowski, M., Guyot, E., Deprez, V., Caplan, D., \& Dominey, P. F. (2003). Training with cognitive sequences improves syntactic comprehension in agrammatic aphasics. NeuroReport, 14, 495-499.

Kazakov, D., \& Bartlett, M. (2004) Co-operative navigation and the faculty of language. Applied Artificial Intelligence, 18, 885-901.

Kirby, S. (2002) Learning, Bottlenecks and the Evolution of Recursive Syntax. In: T. Briscoe (Ed), Linguistic Evolution through Language Acquisition: Formal and Computational Models (pp. 173-203). Cambridge: Cambridge University Press.

Ullman, M. (2004). Contributions of memory circuits to language: the declarative/procedural model. Cognition, 92, 231-270. 